\title{
Laminar Sinusoidal and Pulsatile Flows in a Curved Pipe
}

\author{
M. Jarrahi ${ }^{\dagger}$, C. Castelain and H. Peerhossaini \\ Thermofluids, Complex Flows and Energy Group, LTN - CNRS \\ Ecole Polytechnique-University of Nantes \\ BP 50609, Nantes 44306, France \\ $\dagger$ Corresponding Author Email: mojtaba.jarrahi@univ-nantes.fr
}

(Received April 25, 2010; Accepted March 13, 2011)

\begin{abstract}
Two components of pulsatile flow (i.e. steady flow and sinusoidal flow) are studied separately by particle image velocimetry (PIV). The topology of the secondary flow structures, axial vorticities and transverse strain rates in a pure sinusoidal flow and also in a steady flow are compared to those in a pulsatile flow through a curved pipe. The experimental setup provides different conditions for the flow entering a $90^{\circ}$ circular curved pipe of diameter $0.04 \mathrm{~m}$ and curvature radius $0.22 \mathrm{~m}$. Pulsatile flows were studied for two stationary Reynolds numbers, $\operatorname{Re}_{\mathrm{st}}=420$ and $\operatorname{Re}_{\mathrm{st}}=600$. The frequency parameters $\alpha=10.26$ and $\alpha=14.51$ were chosen to study pure sinusoidal flow $\left(\alpha=r_{0}(\omega / v)^{0.5}\right)$. Pulsating conditions were obtained by combining steady and sinusoidal flow for $\left(\operatorname{Re}_{\mathrm{st}}=600, \alpha=10.26\right),\left(\operatorname{Re}_{\mathrm{st}}=600\right.$, $\alpha=14.51)$ and $\left(\operatorname{Re}_{\mathrm{st}}=420, \alpha=14.51\right)$. The results of this study contribute to a better understanding of mixing in a developing laminar flow through curved pipes (helical and twisted/chaotic mixers) in steady state flow, pure sinusoidal flow and pulsatile flow.
\end{abstract}

Keywords: Curved pipe, Laminar flow, Sinusoidal flow, Pulsatile flow, Mixing, PIV.

$\begin{array}{ll}m & \text { mean value } \\ \max & \text { maximum } \\ P & \text { pulsating flow } \\ r_{0} & \text { pipe cross-sectional radius } \\ r_{C} & \text { curvature radius } \\ \sin & \text { sinusoidal flow } \\ s t & \text { steady flow } \\ t & \text { time } \\ u & \text { velocity in the } x \text { direction } \\ v & \text { velocity in the } y \text { direction } \\ G & \text { gain in mixing process } \\ I & \text { integral of a curve } \\ U & \text { velocity }\end{array}$

\section{INTRODUCTION}

Pulsatile flow in a curved pipe has received considerable attention for many years. The main focus of most previous work on this subject has been biological applications in relation to blood flows (Pedley, 1980; Chang and Tarbell, 1985; Zabielski and Mestel, 1998; Deplano and Siouffi, 1999; Siggers and Waters, 2008). Some other authors have proposed using pulsatile flow through curved tubes in industrial applications such as heat-transfer enhancement (Simon et al. 1977). Only a few studies have looked at using pulsatile flow in curved pipes for mixing intensification (Hydon, 1994; Timité et al. 2009; Jarrahi et al. 2009).

\section{NOMENCLATURE}

$\begin{array}{cc}\operatorname{Re} & \text { Reynolds number, } \operatorname{Re}=\frac{U_{m} D}{v} \\ \alpha & \text { Womersley number, } r_{o}(\omega / v)^{1 / 2} \\ \beta & \text { velocity components ratio } \\ v & \text { kinematic viscosity } \\ \omega & \text { angular frequency } \\ \zeta & \text { axial vorticity, } \frac{\partial v}{\partial x}-\frac{\partial u}{\partial y} \\ \varepsilon & \text { strain rate, } \frac{1}{2}\left(\frac{\partial v}{\partial x}+\frac{\partial u}{\partial y}\right)\end{array}$

The present work extends previous studies by seeking to understand the effects of different flow conditions, i.e. steady state, sinusoidal and pulsatile flow, on transverse mixing variation in a curved pipe.

Mixing in laminar flow regimes is used in many industrial processes. Long molecular chains of viscous fluids will be broken if they are mixed in a turbulent regime. Mixing enhancement in these kinds of problems where the transport mechanism must be carried out slowly needs some new ideas. Benefiting from Dean cell formation in open-loop mixers composed of successive joint curved pipes is one such idea. In this kind of geometry, the problem can be defined as laminar mixing intensification in curved 
pipes. Timite et al. (2009) proposed imposing a temporal dependence on the flow in a curved pipe. Jarrahi et al. (2009) pursued this idea and showed that the pulsating flow can contribute to better laminar mixing in a curved pipe.

In a steady-state flow through a curved pipe, the secondary flow is composed of two counter-rotating cells called Dean cells (Dean 1927). Addition of a pure sinusoidal flow to this steady flow causes a pulsatile flow to form and the structure of the secondary flow becomes much more complex, as shown in the study by Lyne (1971). His analysis of a sinusoidal flow in a curved pipe predicted the appearance of a new vortex pair over and above those observed in the steady case. This new structure, called a Lyne structure, was confirmed analytically by Zalosh and Nelson (1973) and experimentally by Bertelsen (1975). The complexity of the secondary flow increases the capacity of stretching and folding, which are the main mixing mechanisms. Furthermore, using a pulsatile flow for mixing makes it possible to control the process because mixing quality will depend on the oscillation parameters defined by the operator.

Jarrahi et al. (2009) studied the secondary flow structure in pulsatile flows in a curved pipe by PIV measurements for stationary Reynolds numbers $420 \leq \operatorname{Re}_{\mathrm{st}} \leq 1000$, velocity component ratios $1 \leq\left(\beta=\mathrm{U}_{\max , \mathrm{P}} / \mathrm{U}_{\mathrm{m}, \mathrm{st}}\right) \leq 4$ and frequency parameters $8.37<\left(\alpha=\mathrm{r}_{0}(\omega / v) 0.5\right)<24.5$. Comparing the axial vorticities and also the transverse strain rates at the outlet of the curved pipe, they found that in a pulsatile flow only for $\beta \geq 2$ and $\alpha \leq 15$ does the vorticity and strain fields become stronger than those in a steady flow.

The aim of the present work is to better understand the variations in mixing caused by imposing a pure sinusoidal flow on a steady flow. For this reason the secondary flow structures of steady flow and sinusoidal flow are studied separately and then the results are compared to the secondary flow structures of the pulsatile flow produced by the superposition of these steady and sinusoidal flows.

\section{Flow Conditions ANd PARAMETERS}

When analyzing pulsatile flow, it is usual to consider the fluid motion as composed of a steady flow and a pure sinusoidal component. The equation for the pulsatile velocity $\mathrm{U}_{\mathrm{P}}$ can be written as:

$U_{P}(t)=U_{s t}+U_{\text {sin }}(t)$

where $\mathrm{U}_{\mathrm{st}}$ is the mean steady velocity and $\mathrm{U}_{\text {sin }}(\mathrm{t})$ is the pure sinusiodal flow, which is the time-dependent component of the pulsatile velocity. If the amplitude of the sinusoidal flow is $U_{\text {max,sin }}$ and the angular frequency of the oscillation is $\omega$, then Eq. 1 can be written as:

$U_{P}(t)=U_{s t}+U_{\max , \sin } \cdot \sin (\omega . t)$

The angular frequency, $\omega$, can also be described by a dimensionless parameter, $\alpha$, called the Womersley parameter, defined as

$\alpha=r_{0} \cdot(\omega / v)^{\frac{1}{2}}$ $r_{0}$ is the radius of the pipe and $v$ is the kinematic viscosity of the fluid. The Womersley number is a dimensionless expression of the inertial effects due to pulsatile flow frequency in relation to viscous effects and is used in what follows to express the angular frequency of the oscillation.

\section{EXPERIMENTAL FACILITY}

\subsection{Setup}

A volummetric pump connected to a head tank of 300 litre capacity provides the steady component of the flow and an electromagnetic flowmeter measures the flow rate. The sinusoidal component of the flow is generated by a Scotch-yoke mechanism (Fig. 1) in which a piston of $0.04 \mathrm{~m}$ diameter is connected to a crank. The piston stroke is set to $0.06 \mathrm{~m}$ and does not change during the study. A motor speed reducer controls the crank rotational speed and determines the value of the Womersley parameter. The LDV measurements of the axial velocity in previous work (Timité et al. 2009) confirm the reliability of this system in producing a pure sinusoidal flow.

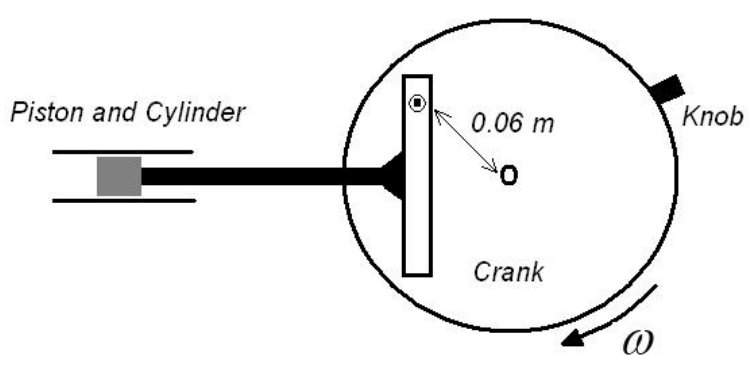

Fig. 1. Scotch-yoke mechanism

The steady part and sinusoidal parts of the flow join at the entrance of a 2.5-meter circular straight tube. This length is sufficient to have a fully developed pulsating flow at the entrance of the curved pipe. The crosssectional diameter of the $90^{\circ}$ curved pipe is $2 \mathrm{r}_{0}=0.04$ $\mathrm{m}$ and the radius of curvature is $\mathrm{r}_{\mathrm{C}}=0.22 \mathrm{~m}$. The straight tube and curved pipe are made of Plexiglas of 5 mm wall thickness.

To eliminate light diffraction effects during PIV measurements, a T-shaped Plexiglas flow divider is installed downstream of the curved pipe. Its outer walls are flat and the cylindrical inner tubes have the same diameter as the curved pipe $(0.04 \mathrm{~m})$. The central branch, which is connected to the curved pipe, is $0.23 \mathrm{~m}$ long and the length of each lateral branch is $0.35 \mathrm{~m}$. The optical window through which the PIV camera observes the illuminated outlet section of the curved pipe is a $3 \mathrm{~mm}$-thick float glass with a multilayer antireflection coating on both sides. Figure 2 gives a schematic presentation of the experimental setup.

\subsection{Measurements}

The particle image velocimetry (PIV) technique, an optical method to obtain instantaneous velocity fields, is used to determine the velocity fields in the secondary flow at the outlet of the curved pipe. A Nd YAG laser 
(50 mJ, $532 \mathrm{~nm}$ ) with sheet thickness $2 \mathrm{~mm}$ and a recording camera $(7 \mathrm{~Hz})$ are used. The camera has a Nikon lens (AF-Micro-NIKKOR 60mm) and is situated in front of the optical window; the laser sheet illuminates the outlet of the curved pipe (Fig. 2). The seeding particles are silver-coated hollow glass spheres (diameter $=10 \mu \mathrm{m}$ ). The resolution of each picture is $2048 \times 2048$ pixels and velocity vectors in the secondary flow are measured by adaptive correlation in the interrogation zones of $64 \times 64$ pixels. A Dantec Dynamics processing system is used to obtain the velocity vectors.

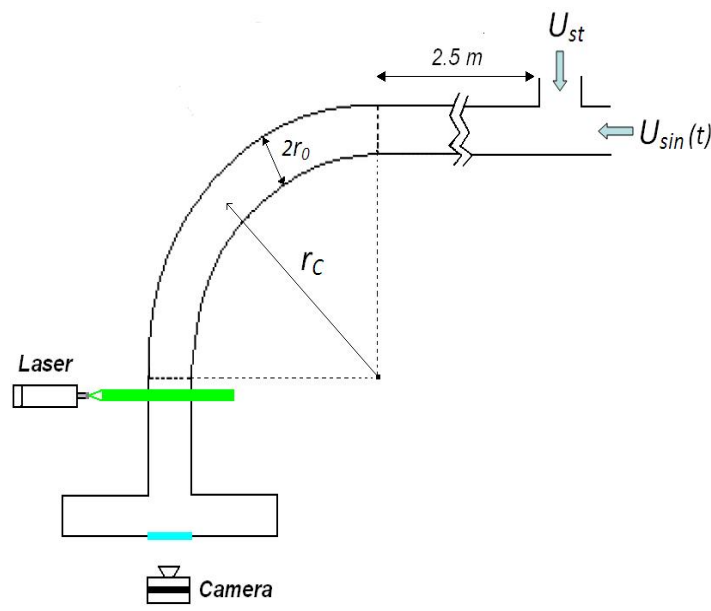

Fig. 2. Experimental setup

Except in the steady-state cases, the measurements for sinusoidal and pulsatile flows are time-dependent and need a synchronization system. Thus the acquisition system is connected to an electric power source controlled by a switching contact device. Each contact of the switch and the small knob fixed on the periphery of the crank sendsa $4.5 \mathrm{~V}$ signal to the acquisition system. The acquisition occurs after a delay that can be calculated based on the desired phase position and the angular velocity of the crank. The measurements have been done in four principal phase positions: $\omega . t=0^{\circ}, 90^{\circ}, 180^{\circ}, 270^{\circ}$. The details of this measurement technique are explained in Jarrahi et al. (2009). In that work, the instantaneous velocity fields for a series of pulsatile flow in the curved pipe are obtained. This study focuses also on the velocity fields of pure sinusoidal flow. An example of the measured velocity fields in four different oscillation phases is given in Fig. 3. Small velocity values are in blue, intermediate values are in yellow and higher values are in red. The left side of this figure shows the velocity vectors of the secondary flow for a pure sinusoidal flow $(\alpha=14.51)$. In the middle of the figure, the velocity vectors in a steady flow $\left(\mathrm{Re}_{\mathrm{st}}=420\right)$ are presented and at the right side, the velocity vectors in a pulsatile flow $\left(\operatorname{Re}_{\mathrm{st}}=420, \beta=2, \alpha=14.51\right)$, which is obtained by superposition of the sinusoidal and the steady flow, are shown.

\section{Results AND Discussion}

As mentioned in the introduction, different structures of secondary flow and mixing in a pulsatile flow through a curved pipe have already been investigated in a previous work by the authors (Jarrahi et al. 2009). It has been shown that a favorable pulsating condition can be obtained when $\beta \geq 2$ and $\alpha \leq 15$. There, the variation in transverse mixing was discussed by comparing the dimensionless vorticities $\left(\left|\zeta_{P}\right| /\left|\zeta_{s t}\right|\right) \quad$ and dimensionless transverse strain rates $\left(\left|\varepsilon_{P}\right| /\left|\varepsilon_{s t}\right|\right)$, where the subscript $\mathrm{P}$ indicates pulsating flow and subscript $\mathrm{S}$ indicates the steady flow with the same Reynolds number. In the present work, the effects of the pure sinusoidal flow component on transverse mixing are studied. $\alpha=14.51$ and $\alpha=10.26$, the Womersely parameters in the range of favourable conditions, $\operatorname{Re}_{\mathrm{st}}=420$ with $\beta=3$ and $\operatorname{Re}_{\mathrm{st}}=600$ with $\beta=1$ and $\beta=2$ have been selected for this study. The velocity fields for these parameters are obtained by PIV at the outlet of the curved pipe, as explained in Section 3.2.

The variations in absolute value of axial vorticity and transverse strain rate during a complete oscillation period for steady flow, sinusoidal flow and pulsatile flow are plotted in Figs. 4 and 5. For each phase position, the average of the absolute values in the section is used as the representative value in that phase position.

For both values of $\alpha$, i.e. $\alpha=10.26$ and $\alpha=14.51$, the maxima of absolute axial vorticity and absolute transverse strain occur near $180^{\circ}$ in pure sinusoidal flows. In pulsatile flow, these maximum values are observed around $90^{\circ}$ when $\alpha=10.26$ (Fig. 3) and around $180^{\circ}$ when $\alpha=14.51$ (Fig. 4). Since the maximum velocity in a sinusoidal oscillation occurs at $90^{\circ}$, these results show that the maximum of absolute axial vorticity and absolute transverse strain values in pure sinusoidal flow take place about $90^{\circ}$ after the velocity maximum.

Adding a steady flow to the sinusoidal flow with $\alpha=10.26$ shows that the maxima of absolute axial vorticity and absolute transverse strain are situated at the same phase position as the when $\alpha$ is increased to 14.51, the phase difference of $90^{\circ}$ is observed again between the maxima of absolute axial vorticity and absolute transverse strain and the velocity maximum. Axial vorticity and transverse strain rate are interesting because they represent folding and stretching, the main mechanisms in mixing. To understand the role of the sinusoidal component of the pulsatile flow in transverse mixing enhancement, two parameters are defined and computed:

$$
\begin{aligned}
& G_{\text {folding }}=\frac{\left(I_{\zeta}\right)_{P}-\left(I_{\zeta}\right)_{\sin }}{\left(I_{\zeta}\right)_{s t}} \\
& G_{\text {stretching }}=\frac{\left(I_{\varepsilon}\right)_{P}-\left(I_{\varepsilon}\right)_{\text {sin }}}{\left(I_{\varepsilon}\right)_{s t}}
\end{aligned}
$$

where $I$ is the integral of a corresponding curve. The subscript $\zeta$ stands for axial vorticity curve; the subscript $\varepsilon$ indicates the transverse strain rate. P" is for pulsatile flow, sin for sinusoidal flow and st for steady flow. $G$ determines the gain in the mixing process resulting from the more complex secondary flow structure obtained by adding a pure sinusoidal flow to a steady flow. In the definition of (4) and (5), the numerator can be interpreted as the net effect on the secondary flow structure of coupling the steady and 

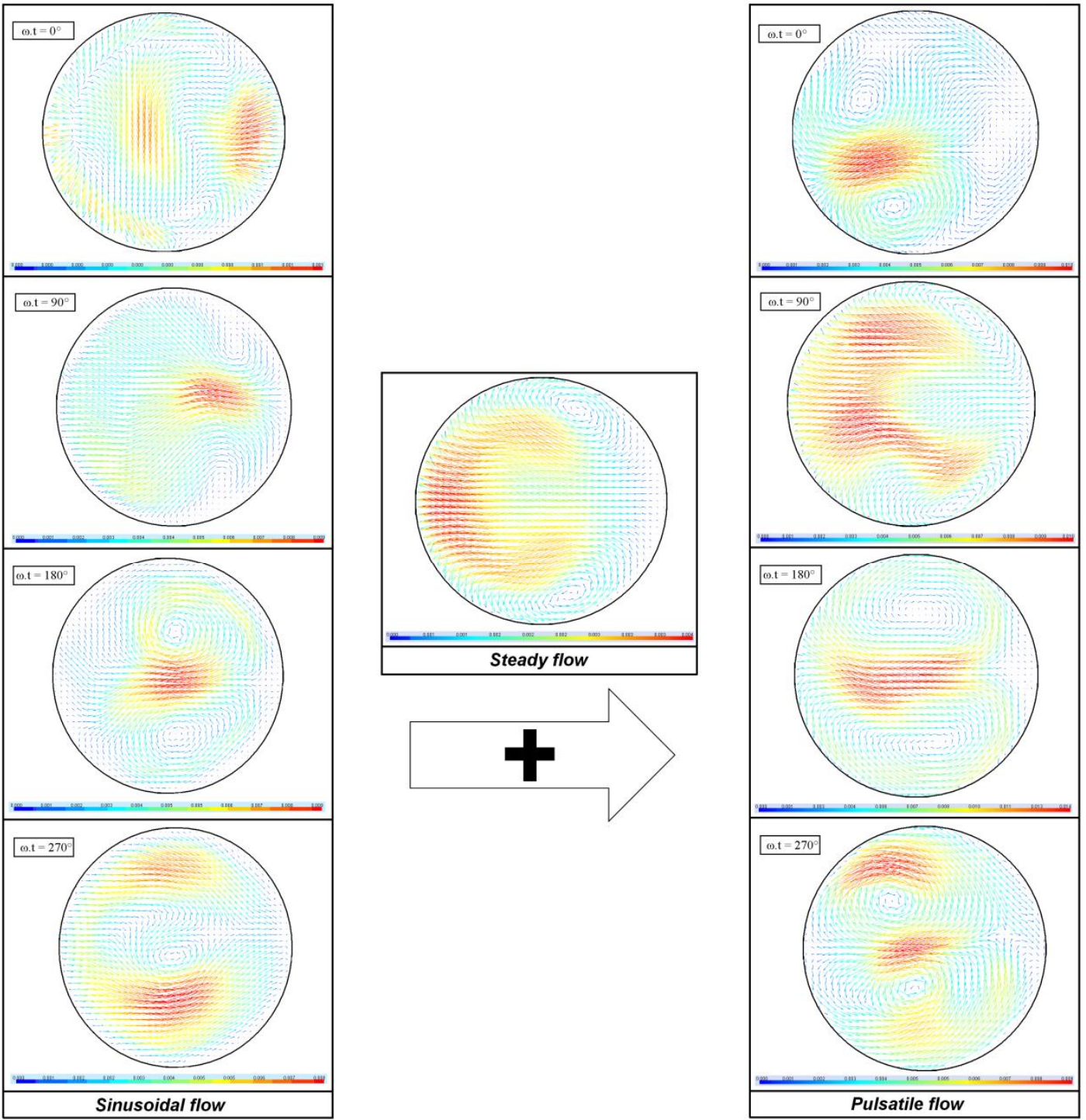

Fig. 3. Velocity vectors of secondary flow in a pure sinusoidal flow $\left(\operatorname{Re}_{\mathrm{st}}=0, \alpha=14.51\right)$, in a steady flow $\left(\operatorname{Re}_{\mathrm{st}}=420\right)$ and in a pulsatile flow $\left(\operatorname{Re}_{\mathrm{st}}=420, \beta=2 \alpha=14.51\right)$ which is obtained by superposition of the sinusoidal and the steady flow.

sinusoidal components, while the denumerator represents the effects of the secondary flow structure only in the steady component of the flow. The different $G$ values can be explained as follows: For a pulsating condition, $G=1$ shows that there is no net effect on the complexity of secondary flow structure compared to the steady condition. $G>1$ for both folding and stretching means that coupling sinusoidal and steady components contributes to the complexity of the secondary flow and it is favorable for mixing; $G<1$ shows that the sinusoidal component of the flow decreases the complexity of the secondary flow structure. The computed values are summarized in Table 1 . The results show that for $\beta=2$ and $\beta=3$ the secondary flow structure, after superposition of the pure sinusoidal flow on the steady flow, is more favorable for mixing than the secondary flow structure of the steady flow.

These results also show that the pure relative gain in mixing by adding a sinusoidal flow to a steady flow is about 2 when $\beta=2$ or $\beta=3$. On the other hand, $\beta=1$ is not strong enough to make a favorable modification in the secondary flow structure. The results of this analysis confirm the previous results of the authors (Jarrahi et al. 2009).

Another index that can show the complexity of the secondary flow structure in a pulsatile flow and its advantages for mixing is the successive formation, displacement, and disapprearance of the elliptic points during an oscillation cycle. The fluid particles turning around an elliptic point are trapped in this zone and cannot be convected to the other zones if the elliptic point does not move (Fig. 6). These zones are considered barriers to mixing because in these regions mass transfer can be done only by diffusion.

When a steady flow passes through a curved pipe, the centers of Dean cells appearing in the structure of the secondary flow are elliptic points.

As remarked above, the regions that surround these points are not useful for mixing. Figure 7 , shows the locus of the center of Dean cells obtained by PIV measurements for $300 \leq \operatorname{Re}_{s t} \leq 1200$ (only the upper 

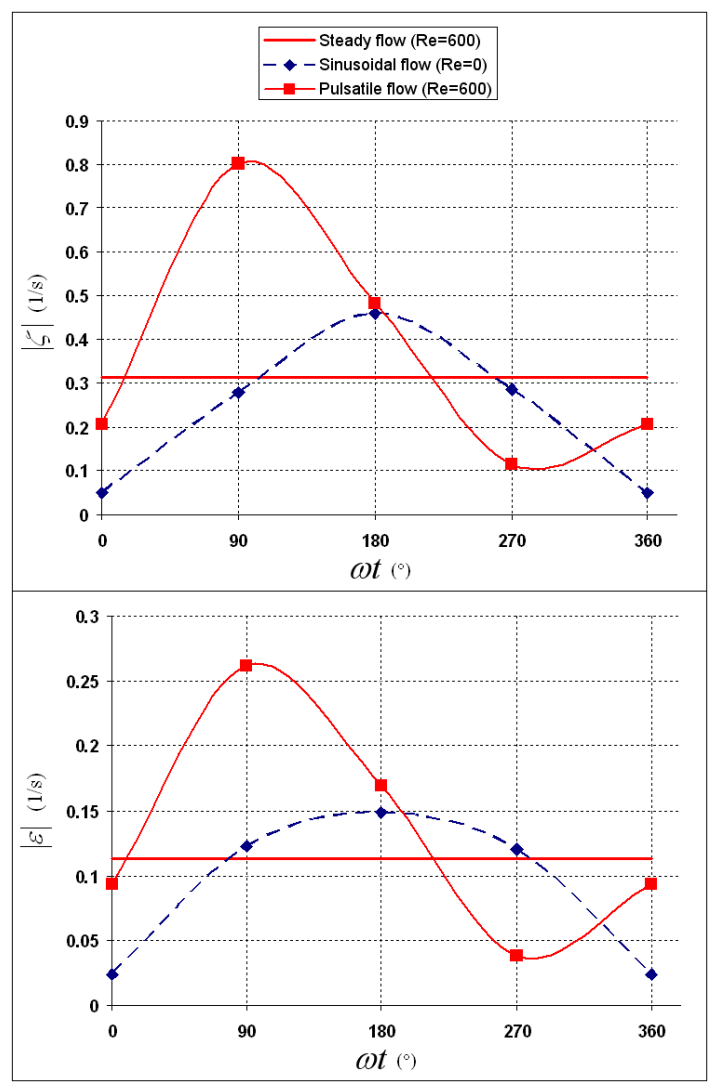

Fig. 4. Variations in absolute values of axial vorticity and transverse strain rate during a complete oscillation period when $\alpha=10.26$.

side of the circular section is shown because the structure of the flow in the steady state is symmetric). Increasing the Reynolds number moves the center of the Dean cells toward the inner and upper sides of the curved pipe.

For a constant Reynolds number in a steady flow, the centers cannot change position because of the stationary nature of the fluid. This kind of elliptic point that remains in the same position is not favorable for mixing because the zones formed around these points extend along the curved pipe and the fluid particles in these regions can hardly leave to mix with the rest of the flow. Unlike in a steady flow, the centers of the secondary flow form, move and disappear successively in a sinusoidal flow. Thus, superposing a sinusoidal flow on the steady flow in the curved pipe breaks up the zones around elliptic points and the displacement of the elliptic points can provide a favorable condition for mixing. Figure 8 shows the movement of the elliptic points in a sinusoidal and a pulsatile flow during a complete oscillation cycle. Here the Womersley parameter is $\alpha=14.51$ and the steady Reynolds number for the pulsatile flow is $\operatorname{Re}_{\mathrm{st}}=600$. No symmetry has been observed and the complex displacement of the elliptic points can be interpreted as a favorable condition for mixing homogeneity because the regions with good mixing and the regions covered by the islands interchange alternately.

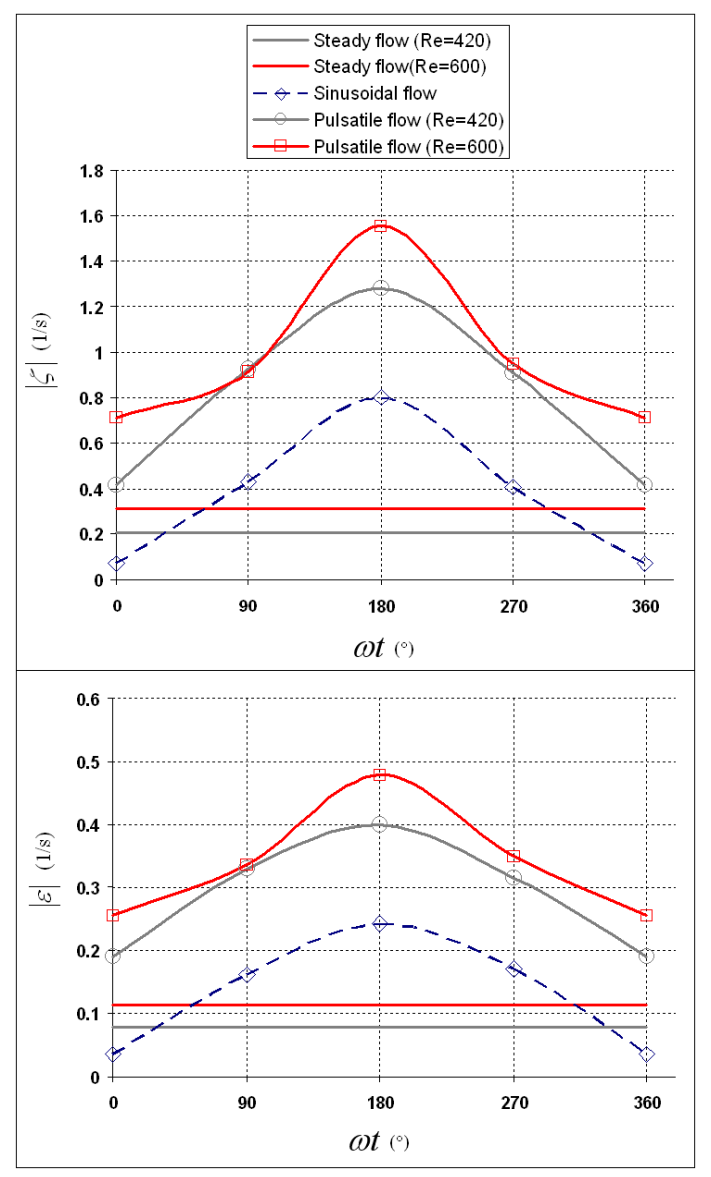

Fig. 5. Variations in absolute values of axial vorticity and transverse strain rate during a complete oscillation period when $\alpha=14.51$.

Table 1. Summary of the calculated Parameters

\begin{tabular}{|c|c|c|}
\hline Flow Condition & \multicolumn{2}{|c|}{ Computed Parameters } \\
\hline $\begin{array}{c}\mathrm{Re}_{\mathrm{st}}=420, \beta=0 \\
\alpha=0 \\
(\text { Steady) }\end{array}$ & $\left(I_{\zeta}\right)_{s t}=74.8$ & $\left(I_{\varepsilon}\right)_{s t}=28.4$ \\
\hline $\begin{array}{c}\mathrm{Re}_{\mathrm{st}}=600, \beta=0, \\
\alpha=0 \\
\text { (Steady) }\end{array}$ & $\left(I_{\zeta}\right)_{s t}=112.7$ & $\left(I_{\varepsilon}\right)_{s t}=40.6$ \\
\hline $\begin{array}{c}\mathrm{Re}_{\mathrm{st}}=0, \alpha=10.26 \\
\text { (Sinusoidal) }\end{array}$ & $\left(I_{\zeta}\right)_{\sin }=96.5$ & $\left(I_{\varepsilon}\right)_{\sin }=37.4$ \\
\hline $\begin{array}{c}\operatorname{Re}_{\mathrm{st}}=0, \alpha=14.51 \\
\text { (Sinusoidal) }\end{array}$ & $\left(I_{\zeta}\right)_{\sin }=154$ & $\left(I_{\varepsilon}\right)_{\sin }=54.9$ \\
\hline \multirow[b]{2}{*}{$\begin{array}{c}\operatorname{Re}_{\mathrm{st}}=420, \beta=3, \\
\alpha=14.51 \\
(\text { Pulsatile })\end{array}$} & $\left(I_{\zeta}\right)_{P}=318$ & $\left(I_{\varepsilon}\right)_{P}=111.3$ \\
\hline & $G_{\text {folding }}=2.2$ & $G_{\text {stretching }}=2$ \\
\hline \multirow[b]{2}{*}{$\begin{array}{c}\operatorname{Re}_{\mathrm{st}}=600, \beta=2, \\
\alpha=10.26 \\
(\text { Pulsatile })\end{array}$} & $\left(I_{\zeta}\right)_{P}=371.4$ & $\left(I_{\varepsilon}\right)_{P}=127.7$ \\
\hline & $G_{\text {folding }}=1.9$ & $G_{\text {stretching }}=1.8$ \\
\hline \multirow[b]{2}{*}{$\begin{array}{c}\mathrm{Re}_{\mathrm{st}}=600, \beta=1 \\
\alpha=14.51 \\
\text { (Pulsatile) }\end{array}$} & $\left(I_{\zeta}\right)_{P}=144.7$ & $\left(I_{\varepsilon}\right)_{P}=50.7$ \\
\hline & $G_{\text {folding }}=0.43$ & $G_{\text {stretching }}=0.33$ \\
\hline
\end{tabular}




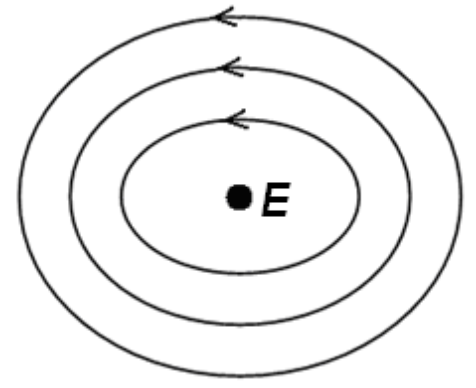

Fig. 6. Elliptic point

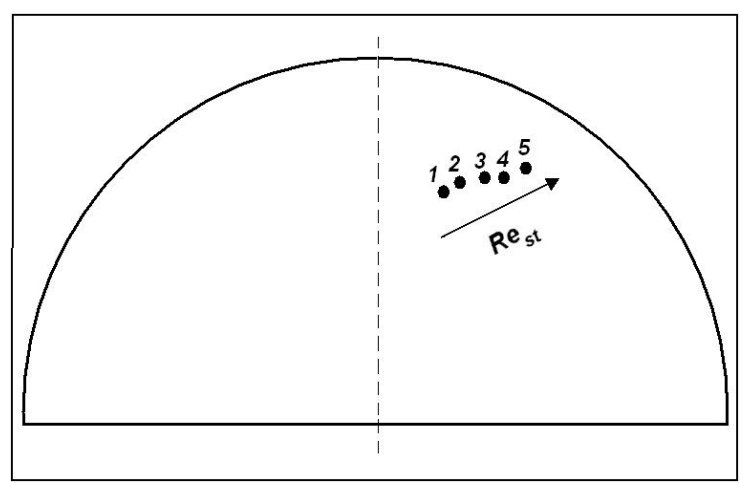

Fig. 7. Locus of center of secondary flow for steady flow when 1) $\operatorname{Re}_{\mathrm{st}}=300$, 2) $\operatorname{Re}_{\mathrm{st}}=420$, 3) $\operatorname{Re}_{\mathrm{st}}=600$, 4) $\left.\operatorname{Re}_{\mathrm{st}}=900,5\right) \operatorname{Re}_{\mathrm{st}}=1200$.

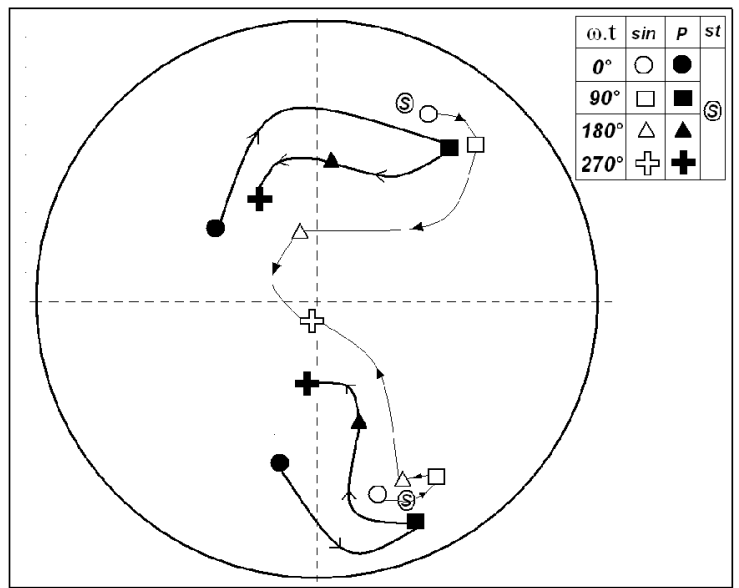

Fig. 8. Locus of center of secondary flow in a cycle when $\alpha=14.51$ for a pure sinusoidal flow (open symbols) and a pulsatile flow with $\mathrm{Re}_{\mathrm{st}}=600$ (filled symbols).

\section{Conclusions}

This study has investigated laminar developing flow through a curved pipe under three different conditions: steady, pure sinusoidal and pulsatile conditions. PIV measurement of the velocity fields in secondary flow allowed analysis of the variation in axial vorticity and transverse strain for different conditions that contributed to the mixing evaluation under different flow conditions. Furthermore, displacement of the centers of cells in the secondary flow (elliptic points) was studied and their complex movement was introduced as another index for mixing enhancement by pulsatile flow.

\section{ACKNOWLEDGEMENTS}

The authors wish to thank CNRS (French National Centre for Scientific Research) for its financial support of this project.

\section{REFERENCES}

Bertelsen, A.F. (1975). An Experimental Investigation of Low Reynolds Number Secondary Streaming Effects Associated With an Oscillating Viscous Flow in a Curved Pipe. Journal of Fluid Mechanics 70, 519-527.

Chang, L.J., and J.M. Tarbell (1985). Numerical simulation of fully developed sinusoidal and pulsatile (physiological) flow in curved tubes. Journal of Fluid Mechanics 161, 175-198.

Dean, W.R. (1927). Note on the Motion of Fluid in a Curved Pipe. Philosophical Magazine 20, 208-223.

Deplano, V., and M. Siouffi (1999). Experimental and numerical study of pulsatile flows through stenosis: wall shear stress analysis. Journal of Biomechanics 32, 10811090 .

Hydon, P.E. (1994). Resonant advection by oscillatory flow in a curved pipe. Physica D. 76, 44-54.

Jarrahi, M., C. Castelain and H. Peerhossaini (2009). Secondary flow velocity field in laminar pulsating flow through curved pipes-PIV measurements. Proceedings of the ASME 2009 Fluids Engineering Division Summer Meeting FEDSM2009, August 2-6, Vail, Colorado USA.

Lyne, W.H., (1971). Unsteady viscous flow in a curved pipe. Journal of Fluid Mechanics 45, 13-31.

Pedley, T.J. (1980). The Fluid Mechanics of Large Blood Vessels. Cambridge Monographs on Mechanics and Applied Mathematics, Cambridge University Press, Cambridge, 160-224.

Siggers, J.H., and S.L. Waters (2008). Unsteady flows in pipes with finite curvature. Journal of Fluid Mechanics 600, 133-165.

Simon, H.A., M.H.Chang, and J.C.F. Chow (1977). Heat transfer in curved tubes with pulsatile, fully developed, laminar flows. ASME Journal of Heat Transfer 99, 590595.

Timité, B., M. Jarrahi, C. Castelain, and H. Peerhossaini (2009). Pulsating flow for mixing intensification in a twisted curved pipe. Journal of Fluids Engineering 131(12), 1104_1-1104_10.

Zabielski, L., and A.J. Mestel (1998). Unsteady blood flow in a helically symmetric pipe. Journal of Fluid Mechanics $370,321-345$.

Zalosh, R.G., and W.G. Nelson (1973). Pulsating flow in a curved tube. Journal of Fluid Mechanics 59, 693-705. 